\title{
VIENNA RADIUM INSTITUTE RADIOCARBON DATES VI
}

\section{HEINZ FELBER}

Institut für Radiumforschung und Kernphysik der Österr Akademie der Wissenschaften, Vienna, Austria

Measurements have continued with the same proportional counter system, pretreatment procedure, methane preparation and measurement, and calculation, as described previously ( $\mathrm{R}, 1970$, v 12, p 298-318). Uncertainties quoted are single standard deviations originating from standard, sample, background counting rates and half-life. No $\mathrm{C}^{13} / \mathrm{C}^{12}$ ratios were measured.

The following list presents most samples of our work in the last year. Sample descriptions have been prepared in cooperation with submitters.

\section{ACKNOWLEDGMENTS}

I express many thanks to Ing L Stein for excellent work in sample preparation, and to A Rasocha for careful operation of the dating equipment.

\section{SAMPLE DESCRIPTIONS}

I. GEOLOGY, GEOGRAPHY, SOIL SCIENCE, AND FORESTRY

\section{A. Austria}

\section{VRI-322. Wallern, Burgenland}

Wood, fragile fragments of oak; depth $7 \mathrm{~m}$, embedded in sand below gravel in subsoil water. Seewinkel between Wallern $\left(47^{\circ} 36^{\prime} \mathrm{N}, 16^{\circ} 56^{\prime} \mathrm{E}\right)$ and Pamhagen, Burgenland. Coll 1971 by Fa Frank, well digger, in Frauenkirchen; subm by H Franz, Hochschule f Bodenkultur, Vienna.

\section{Glacier Pasterze series, Kärnten}

Pressed sandy humus from fossil autochthonous soil below 1 to $2 \mathrm{~m}$ ground moraine. Forefield of glacier Pasterze within lateral moraine from 1856, erosion groove of E Seebach rivulet $\left(47^{\circ} 03^{\prime} 48^{\prime \prime} \mathrm{N}, 12^{\circ} 45^{\prime} 22^{\prime \prime} \mathrm{E}\right)$, Glockner-Group, Hohe Tauern, Carinthia. Site thawed ca 20 yr ago (Patzelt, 1969). Coll 1971 and subm by G Patzelt, Inst Meteorolog Geophys, Univ Innsbruck.

General Comment (GP): samples date passage of advancing glacier over fossil soil. VRI-317 verifies glacier advance proved repeatedly in other areas (Patzelt, 1973). According to stratigraphy dates were expected. No contamination by recent rootlets. Only acid pretreatment was given.

VRI-316. Pasterze 1

Alt $2210 \mathrm{~m}$.

VRI-317. Pasterze 2 Alt 2220m.
$1310 \pm 80$

640 BC

$1700 \pm 100$ AD 250 


\section{VRI-393. Freibach, Kärnten}

Deformed wood remnants in banded sand-clay sediment of former lake probably dammed by mud-flow cone. Site 7 to $10 \mathrm{~m}$ below surface under moraine of former Freibach glacier and gravel. Left border of R Freibach $\left(46^{\circ} 29^{\prime} 18^{\prime \prime} \mathrm{N}, 14^{\circ} 26^{\prime} 47^{\prime \prime} \mathrm{E}\right.$ ) S of bridge Pt 812 (Ö K 1:25000, Part 212/1 Zell Pfarre), Carinthia. Coll 1972 and subm by D van Husen, Inst Geol, TH Vienna.

\section{VR1-396. Großenzersdorf, $N$ ö}

$5690 \pm 100$

Stcm of Elm 10 in terrace gravel of $\mathrm{R}$ Danube dredged at $-5 \mathrm{~m}$ in underground water in gravel pit $2 \mathrm{~km}$ ENE Grossenzersdorf $\left(48^{\circ} 15^{\prime} \mathrm{N}\right.$, $16^{\circ} 35^{\prime} \mathrm{E}$ ), Lower Austria. Coll 1973, subm by J Fink, Geog Inst, Univ Vienna.

\section{VRI-391. Schwarzach, Salzburg}

$13,900 \pm 200$

Wood at base of banded clay several $m$ thick overlying coarse gravel, underlying sand. Artificial opening of R Salzach terrace, Schwarzach $\left(47^{\circ}\right.$ $20^{\circ} \mathrm{N}, 13^{\circ} 10^{\prime} \mathrm{E}$ ), Pongau, Salzburg. Coll 1973 and subm by H Slupetzky, Geog Inst, Univ Salzburg. Comment (HS): 1st date of inner alpine terrace of R Salzach and of ice free period in this region.

\section{Koralpe series, Steiermark}

Peat from bogs of Mt Koralpe, Styria. Coll 1973 and subm by F Kral, Hochschule Bodenkultur, Vienna.

General Comment (FK): establishes chronology of pollen diagram and forest history.

VRI-387. Koralpe 1

Sphagnum peat, bog See-Eben near shelter Stoffhütte $\left(46^{\circ} 53^{\prime} 55^{\prime \prime} \mathrm{N}\right.$, $\left.15^{\circ} 01^{\prime} 25^{\prime \prime} \mathrm{E}\right)$, depth 300 to $310 \mathrm{~cm}$.

\section{VRI-388. Koralpe 2}

Wood peat, bog Filzmoos near Freiländer Alm $\left(46^{\circ} 54^{\prime} 50^{\prime \prime} \mathrm{N}, 15^{\circ}\right.$ $04^{\prime} 10^{\prime \prime} \mathrm{E}$ ), depth 305 to $315 \mathrm{~cm}$.

\section{VRI-392. Bad Aussee, Steiermark}

$11,930 \pm 250$ 9980 BC

Gyttja, base of bog between moraine ramparts. Schmiedgut $\left(47^{\circ} 37^{\prime}\right.$ 15" N, $13^{\circ} 45^{\prime} 50^{\prime \prime}$ E), Bad Aussee, Styria. Coll 1972 and subm by D van Husen. Comment $(\mathrm{DvH})$ : dates climatic deterioration recognized in pollen diagram.

\section{Venter Tal series, Tirol}

Cyperaceae peat from different depths of bog $130 \mathrm{~cm}$ deep near Delorette-Weg $\left(46^{\circ} 49^{\prime} 51^{\prime \prime} \mathrm{N}, 10^{\circ} 49^{\prime} 36^{\prime \prime} \mathrm{E}\right)$, Venter Valley, Ötztaler Alpes, Tyrol, alt $2735 \mathrm{~m}$. Coll 1971 by S Bortenschlager and G Patzelt; 
subm by $S$ Bortenschlager, Inst Bot Systematik Geobot, Univ Innsbruck. General Comment (SB): highest bog of E Alps palynologically analyzed. Observed age inversion probably caused by cryoturbation.

VRI-318. Delorette-Weg 127 to $130 \mathrm{~cm}$

$6790 \pm 140$

Sample from base of bog; depth 127 to $130 \mathrm{~cm}$. Comment (SB): should date beginning of peat growth.

\section{VRI-319. Delorette-Weg 117 to $119 \mathrm{~cm}$}

$7830 \pm 130$

Depth 117 to $119 \mathrm{~cm}$. Comment (SB): sample represents horizon characterized by alternation of peat layers.

\section{VRI-349. Alploachtal, Tirol}

$4990 \pm 100$

Wood from Filzmoos bog, depth $50 \mathrm{~cm}$. Alpbachtal, Lueger Graben, path $S$ of Filzalpe, alt $1640 \mathrm{~m}\left(47^{\circ} 20^{\prime} \mathrm{N}, 12^{\circ} 01^{\prime} \mathrm{E}\right)$, Tyrol. Coll 1971 and subm by $\mathrm{G}$ Mutschlechner, Innsbruck.

\section{VRI-359. Baumkirchen, Tirol}

$27,200 \pm 900$

Wood with roots (Alnus viridis) in undisturbed site in alt $675 \mathrm{~m}$ from banded clay of pit Baumkirchen $\left(47^{\circ} 18^{\prime} 25^{\prime \prime} \mathrm{N}, 11^{\circ} 34^{\prime} 19^{\prime \prime} \mathrm{E}\right)$, Tyrol. Coll 1972 and subm by F Fliri, Geog Inst, Univ Innsbruck. Comment (FF): expected age (Fliri et al, 1970, 1971, 1972; Felber, 1971).

\section{Untergurgl series, Tirol}

Clay gyttja coll by boring from different depths of bog Piller Mösl $\left(46^{\circ} 54^{\prime} 04^{\prime \prime} \mathrm{N}, 11^{\circ} 02^{\prime} 41^{\prime \prime} \mathrm{E}\right)$, alt $1780 \mathrm{~m}$, Untergurgl, Ötztal, Tyrol. Coll by G Patzelt and S Bortenschlager; subm by G Patzelt. Gyttja was extracted with $\mathrm{NaOH}$, precipitated by $\mathrm{HCl}$, and dated.

\section{VRI-365. Piller Mösl, 497 to $500 \mathrm{~cm}$}

$9950 \pm 290$

Depth 497 to $500 \mathrm{~cm}$. Comment (GP): dates beginning of organic sedimentation and 1st recolonization by vegetation. Minimum age of ice retreat in this area.

\section{VRI-366. Piller Mösl 485,5 to $492 \mathrm{~cm}$}

$9520 \pm 220$

Depth 485,5 to $492 \mathrm{~cm}$. Comment (GP): dates palynologically recognized postglacial climatic deterioration.

\section{Imst series, Tirol}

Wood frequently found in present working level of brickyard clay pit Imst $\left(47^{\circ} 13^{\prime} 51^{\prime \prime} \mathrm{N}, 10^{\circ} 45^{\prime} 04^{\prime \prime} \mathrm{E}\right)$, alt ca $730 \mathrm{~m}$, Tyrol. Presumably secondary deposition; originally at least below 4m clay. Coll 1972 and subm by G Patzelt. 
VRI-369. Imst 1

$9890 \pm 150$

7940 BC

Branch (Pinus sp). Comment (GP): dates embedding of forest parts into clay. Age is minimum for ice retreat, clay deposition, and postglacial vegetation development in Imst basin.

VRI-370. Imst 2

$9710 \pm 140$

Stem (Pinus silvestris). Comment (GP): determines contemporaneity of tree embedment.

\section{Matrei series, Osttirol}

Peat cutting Priel near Matrei $\left(46^{\circ} 58^{\prime} 30^{\prime \prime} \mathrm{N}, 12^{\circ} 33^{\prime} \mathrm{E}\right)$, alt $950 \mathrm{~m}$, E Tyrol. Coll 1971 by J Kalhs, subm by F Kral.

General Comment (FK): clarifies period of clearance in Valley of $\mathbf{R}$ Isel shown in pollen diagram.

VRI-336. Matrei 1

AD 1150

$800 \pm 80$

Pine cones and wood remnants (alder?) from depth 55 to $58 \mathrm{~cm}$.

\section{VRI-337. Matrei 2}

$1030 \pm 80$

Dark brown sandy wood peat, possibly contaminated with younger rootiets from depth 58 to $66 \mathrm{~cm}$.

\section{Rostocker Hiitte series, Osttirol}

Sand with fossil humus around shelter Rostocker Hütte $\left(47^{\circ} 03^{\prime} 20^{\prime \prime}\right.$ $\mathrm{N}, 12^{\circ} 18^{\prime} 06^{\prime \prime} \mathrm{E}$ ), alt $2210 \mathrm{~m}$, Maurertal, Venediger Group, E Tyrol, (Patzelt, 1973). Coll 1971 and subm by G Patzelt. Humic acids were extracted, precipitated, and dated.

\section{VRI-367. Rostocker Hütte M-1}

$2030 \pm 80$

Humus overridden by advancing glacier Simonykees and incorporated into moraine $\mathrm{M}$ of this advance. Comment (GP): age is maximum for overriding of fossil soil and older limit for glacier advance see VRI-368.

\section{VRI-368. Rostocker Hütte M-2}

$620 \pm 80$

Humus of soil grown on moraine M of Simonykees before burial by subsequent glacier advances. Comment (GP): age is minimum for underlying moraine, and gives younger limit for deposition of moraine $M$ see VRI-367.

\section{VRI-397. Vienna}

$3210 \pm 90$ $1260 \mathrm{BC}$

Stem wood, oak, embedded in gravel horizon $10 \mathrm{~m}$ thick; near recent R Danube, left bank, km 1922,500, inn "Roter Hiasl" $\left(48^{\circ} 10^{\prime} \mathrm{N}, 16^{\circ} 30^{\prime}\right.$ E), Vienna 22. Position in profile unknown. Excavated by dredging 1973, 
subm by J Fink. Comment (JF): a rounded off Roman brick was found in same stratigraphic location $500 \mathrm{~m}$ upstream at base of this gravel horizon. Thus accumulation of gravel in broad area is quite irregular.

B. Italy, Saudi Arabia, Switzerland, and Spain

\section{VRI-340. Wolfsgruben, Italy}

$12,310 \pm 170$

$5 \mathrm{~cm}$ gyttja on coarse-grained glacial clay overlain by brown moss cyperaceous peat. Base of former lake in quartz-porphyritic depression located in relict pine woodland on Mt Signater Kopf/Ritten, alt $1260 \mathrm{~m}$ $\left(46^{\circ} 31^{\prime} 00^{\prime \prime} \mathrm{N}, 11^{\circ} 25^{\prime} 02^{\prime} \mathrm{E}\right)$ near Wolfsgruben, prov Bozen/Bolzano (Alto Adige), Italy. Coll 1972 by R Schmidt; subm by S Bortenschlager. Comment (HF, RS): overlying $10 \mathrm{~cm}$ peat had to be added for getting enough organic material. No $\mathrm{NaOH}$ pretreatment. Dates beginning of organic sedimentation and late glacial stadial.

\section{VRI-341. Montiggl, Italy}

$12,850 \pm 180$

Lowermost $15 \mathrm{~cm}$ clayey gyttja, $20 \mathrm{~cm}$ thick, overlying clay and underlying brown moss cyperaceous peat, $5 \mathrm{~m}$ thick. Base of former lake in shallow quartz-porphyritic depression near Montiggl $\left(46^{\circ} 25^{\prime} 22^{\prime \prime} \mathrm{N}, 11^{\circ}\right.$ 17' 03" E), alt 495m, prov Bozen/Bolzano (Alto Adige), Italy. Coll 1972 by $\mathrm{R}$ Schmidt, subm by $\mathrm{S}$ Bortenschlager. Comment (RS): dates forest succession in this area.

\section{Langtaufers series, Italy}

Wood from bogs near Langtaufers, N Italy. Coll and subm by G Mutschlechner.

VRI-350. Langtaufers 1

Bog "Moosiges Loch", from $\left.51^{\prime} \mathrm{N}, 14^{\circ} 17^{\prime} \mathrm{E}\right)$. Coll 1971 .

VRI-351. Langtaufers 2 $2170 \mathrm{BC}$

Nameless bog; from $-1 \mathrm{~m}$. Below Kappler See, alt $2520 \mathrm{~m}\left(46^{\circ} 5 \mathrm{l}^{\prime} \mathrm{N}\right.$, $\left.14^{\circ} 16^{\prime} \mathrm{E}\right)$. Coll 1971.

\section{VRI-353. Langtaufers 3}

Small nameless hanging bog, $\mathrm{N}$ above $\mathrm{Melag}$, alt $2070 \mathrm{~m}\left(46^{\circ} 50^{\prime} \mathrm{N}\right.$ 14 $15^{\prime}$ E). Coll 1972.

\section{VRI-352. Graun, Italy}

$4750 \pm 100$

Wood from bog, depth $50 \mathrm{~cm}$. Ochsenberg, SE Hut, alt $2300 \mathrm{~m}\left(46^{\circ}\right.$ $\left.49^{\prime} \mathrm{N}, 14^{\circ} 20^{\prime} \mathrm{E}\right)$, NE Graun, Italy. Coll 1972 and subm by G Mutschlechner. 


\section{VRI-383. Persian Gulf, Saudi Arabia}

$$
1090 \pm 80
$$

AD 860

Shell fossils in horizontal layer $1.3 \mathrm{~m}$ above msl dividing 2 sand dunes of different age. Persian Gulf, W coast $\left(26^{\circ} 30^{\prime} \mathrm{N}, 50^{\circ} 03^{\prime} \mathrm{E}\right)$, Saudi Arabia. Coll 1973 and subm by J Zötl, Inst Min Techn Geol, TH Graz. Comment (JZ): dates old shore line.

\section{VRI-321. Winterthur, Switzerland}

$$
10,930 \pm 160
$$
8980 BC

Wood (Pinus silvestris L) from Trunk 203 of buried Pinus forest, $-7 \mathrm{~m}$ in sand, silt, and clay of cut off "Urstromtal", near Winterthur $\left(47^{\circ}\right.$ $\left.31^{\prime} 15^{\prime \prime} \mathrm{N}, 8^{\circ} 42^{\prime} \mathrm{E}\right)$, Switzerland. Coll 1971 by $\mathrm{F}$ Kaiser; subm by $\mathrm{S}$ Bortenschlager. Comment (SB): dates forest burial.

\section{Tenerife series, Canary Islands, Spain}

Conifer wood under volcanic material. Tenerife, Canary Islands, Spain. Coll by T Bravo, subm by B Schwaighofer, Inst Bodenforschung, Hochschule Bodenkultur, Vienna.

VRI-323. Tenerife 1

Wood in sediments similar to fanglomerate below layer $800 \mathrm{~m}$ thick of alternating basalt and phonolite (Bravo, 1962). Coll 1961. La Guancha, Galeria El Laurel $\left(28^{\circ} 21^{\prime} \mathrm{N}, 12^{\circ} 57^{\prime} \mathrm{E}\right)$. Comment (BS): gives younger limit for embedding sediment.

VRI-324. Tenerife 2

$>36,000$

Wood in clastic material similar Lahar below basaltic layer $400 \mathrm{~m}$ thick of Series III (Bravo, 1962). Coll 1964, Valle de la Orotava $\left(28^{\circ} 20^{\prime}\right.$ $\left.\mathrm{N}, 15^{\circ} 52^{\prime} \mathrm{E}\right)$. Comment (BS): gives younger limit for embedding material.

\section{ARCHAEOLOGIC SAMPLES}

\section{A. Austria}

VRI-300. Nussdorf, O Ö

Modern

Wooden piling, cross section ca $10 \times 10 \mathrm{~cm}^{2}$, from bottom of lake Attersee, Latzl-bay, Nussdorf am Attersee $\left(47^{\circ} 53^{\prime} \mathrm{N}, 13^{\circ} 31^{\prime} \mathrm{E}\right)$, Upper Austria. Coll 1971 by M Reiter, subm by J Reitinger, O Ö Landesmus, Linz. Comment (HF): date disagrees with supposition of Neolithic lake dwelling.

\section{Mooswinki series, Mondsee, $O$ ö}

Soaked remnants of wooden pilings (Picea abies) near shore lifted from bottom of lake Mondsee, $-3 \mathrm{~m}$, Gde Innerschwand, Mooswinkl $\left(47^{\circ}\right.$ $\left.48^{\prime} 50^{\prime \prime} \mathrm{N}, 13^{\circ} 23^{\prime} 40^{\prime \prime} \mathrm{E}\right), \mathrm{O}$ Ö. Coll 1972 and subm by J Offenberger, Bundesdenkmalamt, Wien.

General Comment (JO): dates prove Neolithic lake dwellings ( $R, 1973$, v 15, p 433). 
VRI-331. Mooswinkl 3

$4350 \pm 90$

VRI-332. Mooswinkl 4

2400 BC

$4260 \pm 90$

VRI-333. Mooswinkl 5

2310 BC

$4430 \pm 110$

2480 BC

\section{Hallein series, Salzburg}

Wood fragments of fire sticks, props and tools in different parts of prehistoric salt mine Dürrnberg near Hallein (Schauberger, 1968) (47\% $\left.39^{\prime} 30^{\prime \prime} \mathrm{N}, 13^{\circ} 05^{\prime} \mathrm{E}\right)$, Salzburg. Subm 1970 by O Schauberger, Bad Ischl, O Ö.

\section{VRI-268. Central group, 3/1}

$1890 \pm 90$

Fire sticks in "laistigem Heidengebirge" (deads of rock salt in form of plastic saliferous clay), S part of Central group, Georgenberg-horizon, Querschlag III, 80m from Wechsel, Site 3. Coll 1959 by O Schauberger.

\section{VRI-269. Central group, 12a}

$2000 \pm 80$

Fire sticks and charcoal in "kernigem Heidengebirge" (deads of rock salt; salt fragments cemented to breccia by saliferous clay), W of Central group, Obersteinberg-horizon, Ferro-Schachtricht, Site 12a. Coll 1958 by O Schauberger.

VRI-288. Central group, $3 / 2$

$2300 \pm 90$

Tool in "laistigem Heidengebirge", S of Central group, Georgenberghorizon, Site 3 . Coll 1970 by A Aschauer.

VRI-289. Central group, 13a

$2420 \pm 90$

$470 \mathrm{BC}$ Aschauer.

Prop, W of Central group, Werk O/9, Site 13a. Coll 1970 by A

VRI-290. Central group, 5

$2670 \pm 80$

720 BC

Tool in "Heidengebirge", Central group, Georgenberg-horizon, Werk Platz, Site 5. Coll 1971 by A Aschauer.

VRI-291. S group, 1

$$
2090 \pm 80
$$

Fragment of prop in "kernigem Heidengebirge", $\mathbf{S}$ group, Kelbhorizon, Werk Schrempf, Site 1. Coll 1950 by O Schauberger.

VRI-292. S group, 3a

Fragment of prop in "kernigem Heidengebirge", $S$ group, Georgenberg-horizon, Werk Brandner, Site 3a. Coll 1967 by O Schauberger. 
VRI-293. S group, 3b

Fragment of prop in "kernigem Heidengebirge", S group, Georgenberg-horizon, Werk Mitterauer, Site 3b. Coll 1970 by A Aschauer.

\section{B. Greece, Turkey}

\section{VRI-395. Aegina, Greece}

Charcoal from fortification of ancient Aegina, Aegina I. $\left(37^{\circ} 45^{\prime} \mathrm{N}\right.$, $23^{\circ} 25^{\prime} \mathrm{E}$ ), near Athens, Greece. Coll 1972 and subm by H Walter, Inst Klass Archäol, Univ Salzburg. Comment (HW): sample from habitation level of early Bronze age in 3rd millennium BC. Dates destruction of fortification. De Vries corrected date, 2100 BC, fits archaeologically determined age (Weinberg, 1967).

\section{VRI-329. Ephesos, Turkey}

$2390 \pm 80$

Burnt remnants of wood $3 \mathrm{~m}$ below loamy horizon excavated with pottery and bones in area between altar and temple of Diana (Bammer, 1972; Vetters, 1973) in Ephesos (37 $57^{\prime} \mathrm{N}, 27^{\circ} 20^{\prime} 10^{\prime \prime} \mathrm{E}$ ), Turkey. Coll 1971 and subm by A Bammer, Österr Archäol Inst, Univ Vienna.

\section{REFERENCES}

Bammer, A, 1972, The Altar of Artemis at Ephesus: The annual of the ruins and museum of Ephesus 1972, p 76-82.

Bravo, T, 1962, El circo de las Cañadas y sus dependencias: Bol R Soc Esp Hist Nat (G), v 60, p 93-108.

Felber, H, 1971, Altersbestimmungen nach der Radiokohlenstoffmethode an Fossilfunden aus dem Bänderton von Baumkirchen (Inntal, Tirol): Gletscherkde Glazialgeol Zeitschr, v 7, p 25-29.

Fliri, F et al, 1970, Der Bänderton von Baumkirchen (Inntal, Tirol) eine neue Schlüsselstelle zur Kenntnis der Würmvereisung der Alpen: Gletscherkde Glazialgeol Zeitschr, v 6, p 5-35.

Fliri, F, Felber, H, and Hilscher, H, 1972, Weitere Ergebnisse der Forschung am Bänderton von Baumkirchen (Inntal, Nordtirol): Gletscherkde Glazialgeol Zeitschr, v 8, p 203-213.

Fliri, F, Hilscher, H, and Markgraf, V, 1971, Weitere Untersuchungen zur Chronologie der alpinen Vereisung (Bänderton von Baumkirchen, Inntal, Nordtirol): Gletscherkde Glazialgeol Zeitschr, v 7, p 5-24.

Patzelt, G, 1969, Zur Geschichte der Pasterzenschwankungen; Neue Forschungen im Umkreis der Glocknergruppe: Wiss Alpenvereinshefte no. 21, Wien, p 171-179.

1973, Die postglazialen Gletscher- und Klimaschwankungen in der Venedigergruppe (Hohe Tauern, Ostalpen): Z Geomorph NF, Berlin, Stuttgart, Supp v 16, p 25-72.

Schauberger, O, 1968, Die vorgeschichtlichen Grubenbaue im Salzberg Dürrnberg/ Hallein: Prähist Forschungen, no. 6, Anthropol Gesell Wien.

Vetters, H, 1973, Ephesos. Vorläufiger Grabungsbericht 1972: Anz Österr Akad Wiss Wien, phil-hist Kl, Jahrg 1973, p 176-194.

Weinberg, 1967, The relative chronology of the Aegean, in: Ehrich R, 1967, Chronologies in Old World archaeology: Chicago-London. 